\title{
Final Report for LDRD Project on Rapid Problem Setup for Mesh-Based Simulation (Rapsodi)
}

D. L. Brown, W. Henshaw, N. A. Petersson, P. Fast, and K. Chand

February 7, 2003

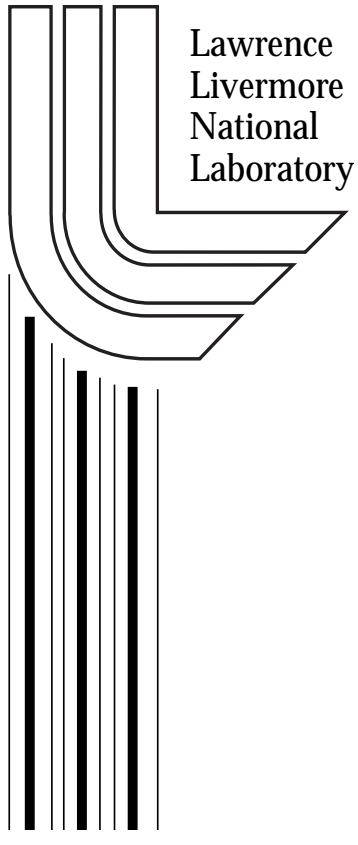




\section{DISCLAIMER}

This document was prepared as an account of work sponsored by an agency of the United States Government. Neither the United States Government nor the University of California nor any of their employees, makes any warranty, express or implied, or assumes any legal liability or responsibility for the accuracy, completeness, or usefulness of any information, apparatus, product, or process disclosed, or represents that its use would not infringe privately owned rights. Reference herein to any specific commercial product, process, or service by trade name, trademark, manufacturer, or otherwise, does not necessarily constitute or imply its endorsement, recommendation, or favoring by the United States Government or the University of California. The views and opinions of authors expressed herein do not necessarily state or reflect those of the United States Government or the University of California, and shall not be used for advertising or product endorsement purposes.

This work was performed under the auspices of the U. S. Department of Energy by the University of California, Lawrence Livermore National Laboratory under Contract No. W-7405-Eng-48.

This report has been reproduced directly from the best available copy.

Available electronically at http://www.doc.gov/bridge

Available for a processing fee to U.S. Department of Energy

And its contractors in paper from

U.S. Department of Energy

Office of Scientific and Technical Information

P.O. Box 62

Oak Ridge, TN 37831-0062

Telephone: (865) 576-8401

Facsimile: (865) 576-5728

E-mail: reports@adonis.osti.gov

Available for the sale to the public from

U.S. Department of Commerce

National Technical Information Service

5285 Port Royal Road

Springfield, VA 22161

Telephone: (800) 553-6847

Facsimile: (703) 605-6900

E-mail: orders@ntis.fedworld.gov

Online ordering: http://www.ntis.gov/ordering.htm

OR

Lawrence Livermore National Laboratory

Technical Information Department's Digital Library

http://www.llnl.gov/tid/Library.html 
Final Report for LDRD Project on

\title{
Rapid Problem Setup for Mesh-Based Simulation (Rapsodi)
}

\author{
Tracking Code: 00-ERD-017
}

\author{
David L. Brown, PI \\ dlb@1lnl.gov \\ William Henshaw \\ N. Anders Petersson \\ Petri Fast \\ Kyle Chand \\ Computer Applications Research Department \\ Computation Directorate
}

\begin{abstract}
Under LLNL Exploratory Research LDRD funding, the Rapsodi project developed rapid setup technology for computational physics and engineering problems that require computational representations of complex geometry. Many simulation projects at LLNL involve the solution of partial differential equations in complex 3-D geometries. A significant bottleneck in carrying out these simulations arises in converting some specification of a geometry, such as a computeraided design (CAD) drawing to a computationally appropriate 3-D mesh that can be used for simulation and analysis. Even using state-of-the-art mesh generation software, this problem setup step typically has required weeks or months, which is often much longer than required to carry out the computational simulation itself. The Rapsodi project built computational tools and designed algorithms that help to significantly reduce this setup time to less than a day for many realistic problems. The project targeted rapid setup technology for computational physics and engineering problems that use mixed-element unstructured meshes, overset meshes or Cartesianembedded boundary (EB) meshes to represent complex geometry. It also built tools that aid in constructing computational representations of geometry for problems that do not require a mesh. While completely automatic mesh generation is extremely difficult, the amount of manual labor required can be significantly reduced. By developing novel, automated, component-based mesh construction procedures and automated CAD geometry repair and cleanup tools, Rapsodi has significantly reduced the amount of hand crafting required to generate geometry and meshes for scientific simulation codes.
\end{abstract}




\section{PROJECT OVERVIEW}

The Rapsodi project addressed the problem set-up needs of Laboratory and DOE simulation and analysis codes through a new approach for generating unstructured mixed-element meshes and through the development of automated techniques for cleanup and repair of initial geometry specifications. The repair and cleanup tools are applicable to all mesh generation types, and hence to all Laboratory applications that require a geometry representation or computational mesh. The unstructured mesh generation capability was specifically targeted at those applications that have the ability and need to use mixed-element or tetrahedral meshes, such as the KULL project in A division and the EMSolve project in Engineering.

Typically, the initial geometric information provided for a simulation consists of a detailed engineering specification given by a Computer-Aided Design (CAD) package. Commonly, this information is provided to the mesh generator as a surface representation through the common file format in an IGES (Initial Graphics Exchange Specifications) file. Unfortunately, these representations are often inconsistent and incomplete, or even overly detailed. Repairing and cleaning up the surface representations, known as the "CAD healing" problem, can often consume a large fraction of the total effort in generating a mesh. For example, the Boeing Corporation estimates that it spends one quarter of its mesh generation time and around $\$ 100 \mathrm{M}$ per year on this step alone ${ }^{1}$. The Rapsodi project has developed new tools that address CAD healing, and incorporate those tools directly within meshing software for generating mixed element, overset and embedded boundary Cartesian meshes.

A second issue is that for the mesh generation process to be sufficiently fast, it must be possible to rapidly evaluate the surface and topology of the geometry, particularly in cases where the mesh generator needs to be called from within an application for adaptive mesh refinement purposes, or in the case of moving geometry. Rapsodi has developed tools that enable the rapid evaluation of the trimmed NURBS (non-uniform rational B-spline) surface representations that are provided by the CAD package.

Rapsodi has also developed an automated component-based-mesh generation (CBM) procedure for rapidly generating high-quality unstructured "hybrid" or "mixed element" meshes that contain large regions of structured (hexahedral) cells. In this approach, a set of structured "component" meshes is created whose union nearly covers the computational region. These component meshes are then connected with regions of mixed-element unstructured mesh. CBM meshes represent a completely new approach to mesh generation for the mainstream Laboratory applications, and provide new flexibility in the ability to generate meshes for an important subset of the major Laboratory application codes.

Rapsodi has developed new algorithms and software for decomposing CAD-based geometry into mesh-able components. We have also developed robust algorithms for generating these connection meshes in two and three dimensions. The construction of high-quality structured component meshes for mapped geometries is an important step in this process. To support this step, we have developed improved marching algorithms for "growing" surface meshes on CAD surfaces and also three-dimensional volume meshes. The marching process involves the solution 
of hyperbolic partial differential equations combined with mesh smoothing algorithms. A particularly important feature of the CBM procedure is that it is incremental. New components can be added to an existing CBM mesh without completely regenerating the original mesh.

Evaluating and optimizing the quality of a mesh for a given computation requires understanding how that mesh will perform with respect to a given simulation algorithm. Rapsodi began the development of tools for the optimization of hybrid meshes based on metrics that involve the quality of simulation solutions on those meshes.

\section{ACCOMPLISHMENTS}

The problem set-up process consists of all the steps that must be taken to convert a specification of geometry to a computationally-appropriate $3 \mathrm{D}$ representation that can be used for simulation and analysis. In many cases, this representation is a 3D volumetric mesh. This project focused on the CAD geometry repair and mesh generation parts of this process. Currently, using state-ofthe-art mesh generation software, it often takes simulation scientists at the Laboratory and in industry weeks or months to generate a new computational mesh from scratch. The objective of this project was to develop algorithms and tools that would help reduce this setup time to under a day.

In this project, we focused on the case when the initial geometric information consists of a detailed engineering specification given by a Computer-Aided Design (CAD) package. We identified two research strategies that were effective in significantly improving the turn-around time for the mesh-generation process. These were the development of improved techniques for $\mathrm{CAD}$ cleanup and repair, and the development of a new technique for generating high-quality mixed-element meshes.

The mixed-element mesh generation process requires a good quality geometrical description that can be easily evaluated and queried. (In fact, this is true for any mesh generation technique). Given this geometry description, a variety of tools for generating high-quality component meshes are required. The component meshes must then be connected together to form a consistent representation of the entire region of interest. Rapsodi was able to build tools for generating mixed-element hybrid meshes by leveraging the mesh generation infrastructure and capabilities available through the Overture framework at the Center for Applied Scientific Computing (CASC) at the Laboratory. Overture brought significant infrastructure to Rapsodi for dealing with imported 3-D CAD descriptions, generating component meshes using a variety of techniques and for visualization and graphical editing of geometrical information ${ }^{2,3}$.

\section{A. CAD Cleanup and Repair}

Rapsodi interacts with CAD descriptions through a common file format called IGES (Initial Graphics Exchange Specifications). Since most common CAD packages can produce this type of output file, our methods apply to data generated by many CAD packages. The representation we extract from an IGES file is a set of trimmed NURBS (figure 1). These consist of trimmed spline patches that completely cover the surface. This representation is supposed to be "watertight" and single-valued, although as a side-effect of the fact that the CAD representation is not 
originally created with mesh-based simulation and analysis tools in mind, this is often not the case. Converting the input geometry to a mesh-appropriate representation can include repairing the CAD by filling gaps, reconciling errors in NURBS trimming curves, determining NURBS surface connectivity information, and removing unnecessary engineering details from the specification. The tools we developed allow much of this work to be done automatically; for the aspects that cannot be completely automated, we provided an interactive graphics interface to tools for surface repair and improvement. Under this LDRD project, we developed an
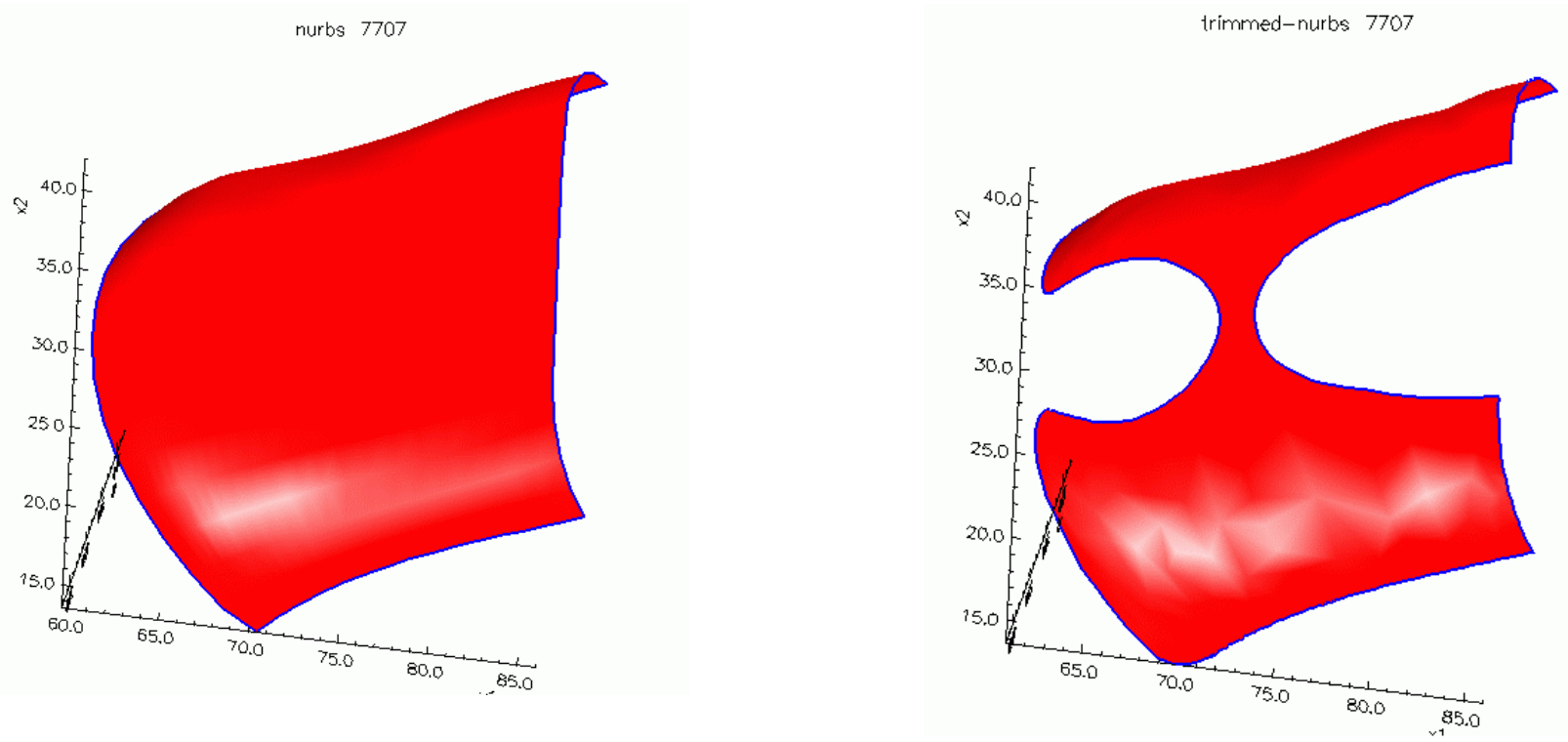

Figure 1. A NURBS representation uses splines to represent a surface patch (left). Trimming curves are added to the description to provide more detail (right). Errors in the trimming curves result in an inconsistent CAD geometry description.

interactive graphics tool called "RAP" for the cleanup and repair of CAD surfaces. ${ }^{4,5}$ In investigating a number of complex CAD descriptions in IGES file format, we discovered that a very common source of errors lies in the trimming curve descriptions for the trimmed NURBS representation. A missing trimming curve segment, for example, results in an indeterminate description for the associated segment of the geometry, a situation that must be resolved. In our software tool, these indeterminate patches are automatically detected. As part of this process, the connectivity information for the NURBS patches is automatically determined by the software. Tools are provided to help the user interactively correct the description. Typically this will involve adding missing sections of the trimming curve, remove unneeded geometrical features, and patch resulting holes in the description. We have tested this tool using a CAD geometry provided by the Volvo Car Corporation that describes an automobile engine intake manifold. Of the approximately 350 patches in the description, about 50 faulty patches were detected and repaired. An additional 30 patches that described components of the geometry not needed for a computational fluid dynamics simulation were removed and the resulting holes were filled. The total cleanup and repair process for this example takes on the order of an hour using our new software tool (figure 6). 


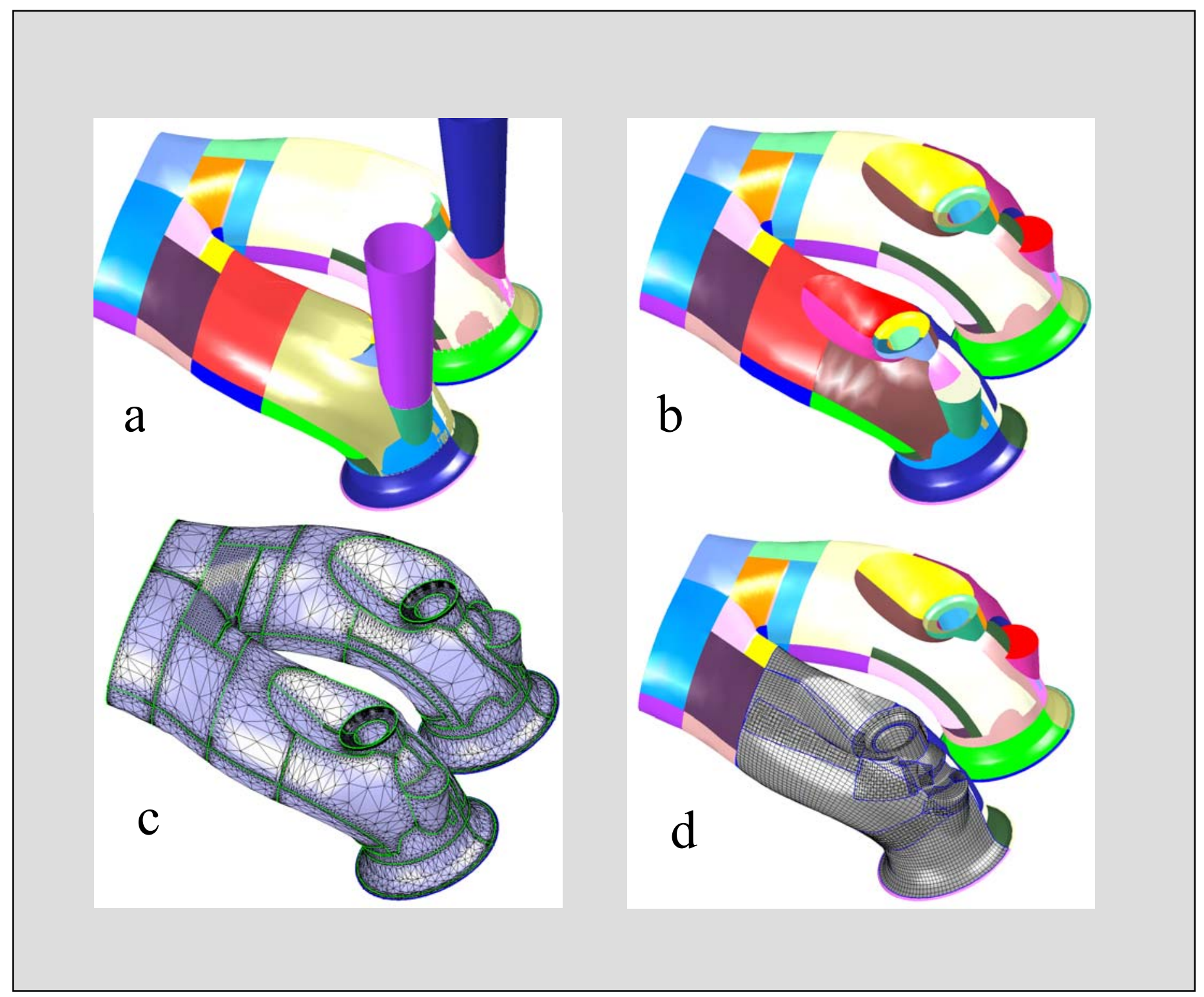

Figure 2. This figure show many of the steps in the automated CAD-to-mesh process. An original geometry (a) represented by a set of NURBS patches is repaired and modified to produce a preliminary geometry for meshing (b). Connectivity information for the resulting repaired surface patches, and a data structure that enables rapid interrogation of the surface is then constructed (c). The surface patch information plus data structures is then used as by the surface mesh generation procedures to cover the surface with a composite overlapping set of surface meshes (d). Further steps in the process involve the generation of volume meshes using the surface meshes as starting surfaces.

\section{B. Rapid Evaluation of CAD surfaces; automatic partitioning of geometry}

Once the CAD geometry has been cleaned up and repaired, it can be used to generate a computational mesh. We use information from the NURBS representation to subdivide the surface representation into components that are then individually meshed. As a practical matter, we require that the component meshes be generated within seconds on a typical scientific 
workstation; this necessitated the development of very fast algorithms for the point evaluation step. Thus, the research issues here were (1) the development of algorithms for automatically partitioning surfaces, and (2) algorithms for rapidly evaluating the location of points on the NURBS surface.

The surface meshes are built using hyperbolic mesh generation, so named because the meshes are determined by numerically solving a hyperbolic or near-hyperbolic partial differential equation; starting from a curve on the surface, a mesh is grown by marching over the surface. A given hyperbolic grid will typically cover many of the underlying CAD surface patches. The essential operation needed for building surface grids is the operation of projecting a point in space onto the closest point on the CAD surface. We have developed a fast algorithm for doing this that makes use of a fairly coarse global triangulation of the CAD geometry.

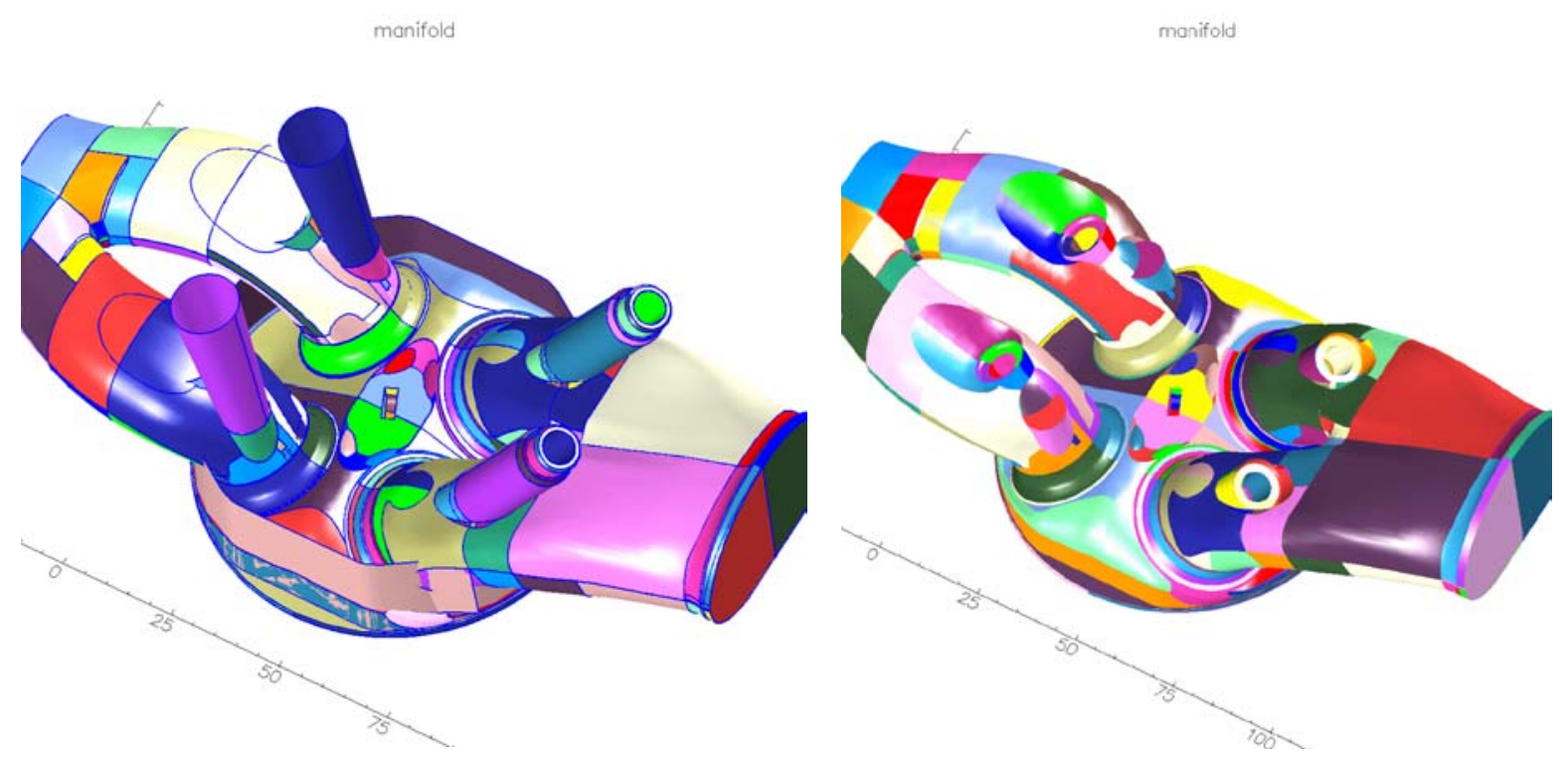

Figure 3. Volvo intake manifold original CAD geometry (left) and "healed" geometry (right).

Rapidly evaluating a trimmed NURBS surface can be difficult. The IGES geometry file contains a list of these NURBS patches, but no information about adjacency or orientation of the patches. Since the representations are in terms of polynomials in three-space, but the trimming curves are defined in a two-dimensional parameter space, the edges of adjacent patches do not exactly align, even though they may appear to. Our approach is to automatically determine the adjacency information, and to resolve inconsistencies in adjacent edge descriptions. We then construct a triangulation of the surface (see figure 2). This triangulation is not used for computation, but actually becomes a data structure that helps speed up the evaluation of surface points. When it is necessary to evaluate a point on the surface, the corresponding location is determined on the triangularization (a much quicker process), and then information stored with the triangularization is used to determine the original location on the patched NURBS surface.

In order to build this triangulation, we first determine the connectivity of the CAD surface patches (information not available in the IGES file). This is done by building curves on the 


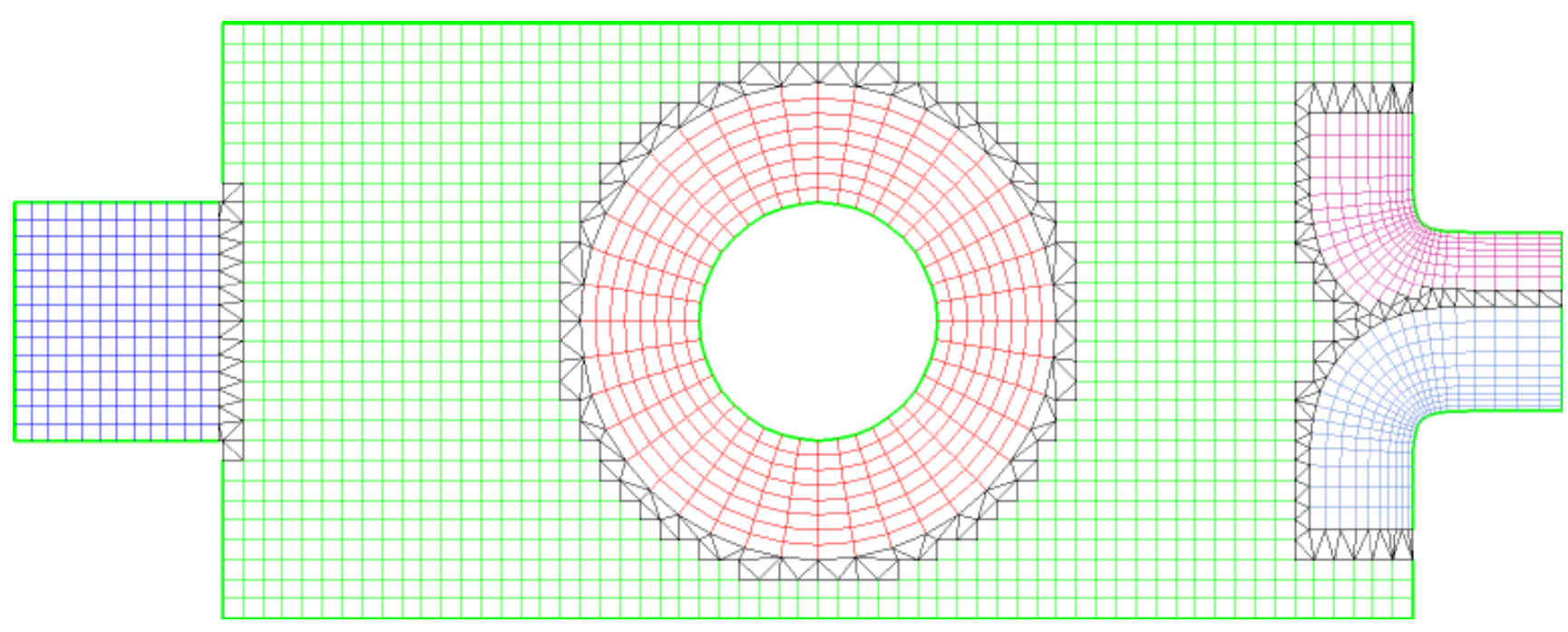

Figure 4. A hybrid mixed mesh connects large areas of structured mesh using local unstructured regions.

boundaries of all trimmed patches and then identifying where an edge-curve from one patch matches to the edge-curve of a neighboring patch. This connection algorithm has the side benefit that it provides an effective way to detect errors in the CAD representation such as overlaps or gaps between adjacent patches. Once the edge-curves have been matched, a global triangulation is formed for the patched surface. This is done by first distributing node points on the edges of each patch and then projecting those nodes to the parameter space of each patch, where the triangulation is done. All nodes of the triangulation are then mapped back to three-space. Since the node point distribution is the same along edges shared by two patches, the resulting triangulation will share nodes along these edges by construction (see figure 2, above). This results in a fast algorithm in which the triangulation is done in the two-dimensional parameter space rather than on the three-dimensional surface. This global triangulation serves as a basis for a fast projection algorithm for projecting points onto the patched surface. Using this new approach together with new optimized NURBS evaluation routines has enabled us to speed up the generation of surface meshes by a factor of 100 from our original prototype implementation. The result is that the surface mesh generation code is now a highly interactive procedure (figure 3). ${ }^{6}$

Part of the hybrid mesh generation process is the componentization of surface descriptions before generating component surface meshes. Information in the NURBS representation can often be used to automatically determine, or "suggest" appropriate starting curves for the hyperbolic mesh generation process. For example, edges can be detected automatically and suggested as starting curves. We classify edges as those interior to the surface and those that lie on the boundary of the surface. Edges on the boundary are automatically joined (provided they meet in a smooth way) to form smooth boundary curves. These boundary curves are often used as starting curves for surface grids. The user may interactively choose a sequence of edges from the interior or boundary and these will be concatenated to form a curve that can also be used as a starting curve. 


\section{Hybrid Mesh Generation}

In our hybrid mesh generation approach, a high-quality structured grid is first generated for each component in a geometry (see section $\mathrm{D}$, below). Once the volume meshes describing each of

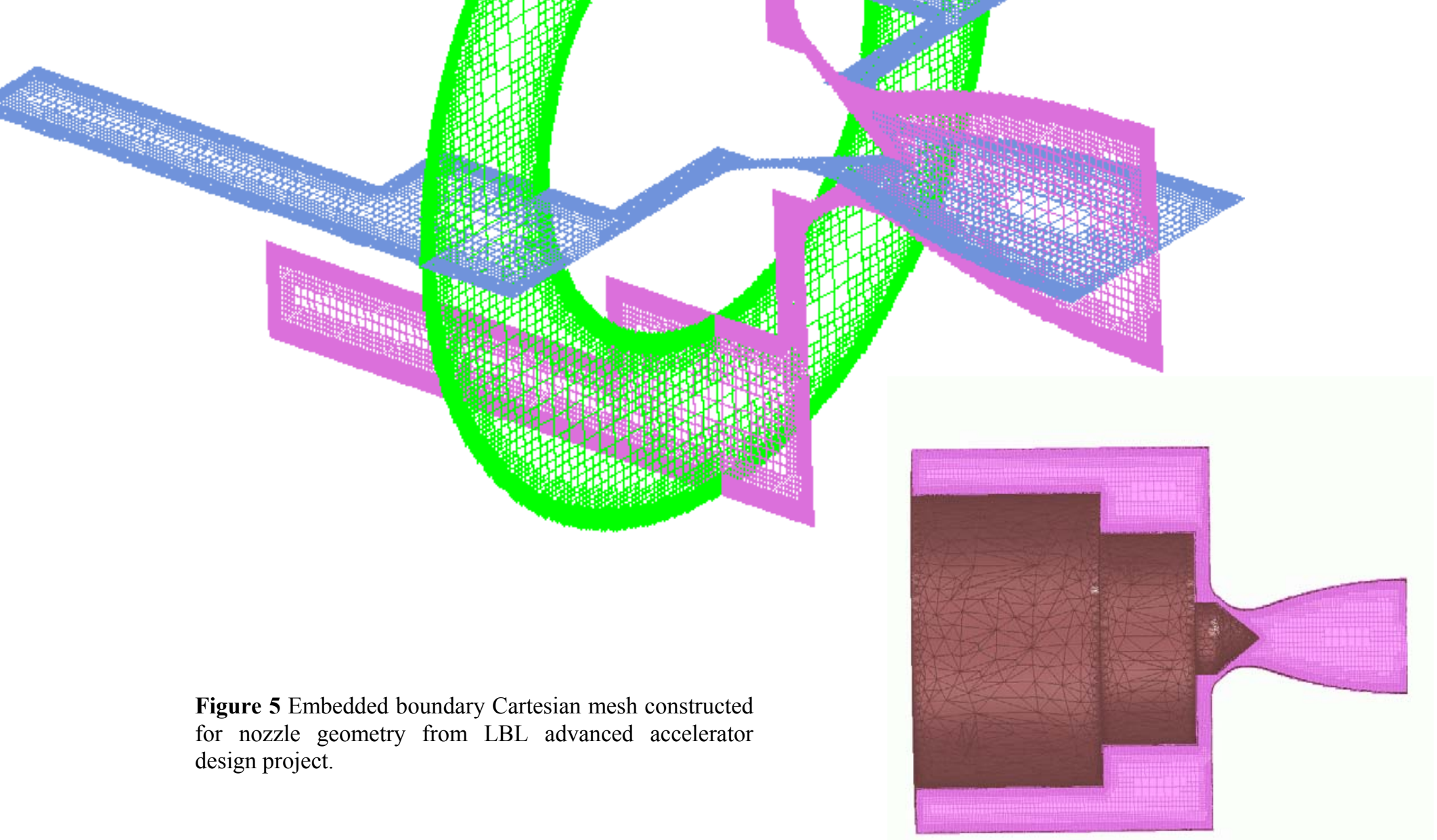


the sub-components of a geometry have been generated, the hybrid mesh is completed by cutting holes in some meshes where they overlap, and generating unstructured mesh regions to connect the component meshes. The Ogen ${ }^{2}$ mesh generator from the Overture project is used to detect the mesh overlap and remove it, leaving an unmeshed hole in the geometry. An advancing front approach $^{7}$ is used to generate a high-quality mesh to fill the resulting connection regions. Triangles are used to construct the transition mesh in two dimensions; in three dimensions, the transition mesh starts with pyramids on the structured mesh interface, and fills the remaining space with tetrahedra. In order to maintain the quality of the unstructured mesh, we have implemented a mesh stretching control procedure that attempts to guarantee that the unstructured mesh cells are well matched to the structured cells on either side of the transition region. These algorithms have been implemented within the Ogen mesh generator, and have required the development of appropriate data structures for storage and optimization of the algorithms. ${ }^{8}$ The hybrid mesh generation algorithms are described in more detail in a paper and in published code documentation. $9,10,11$ The end result at this step is an unstructured mesh over which the user has had considerable control, and with large regions of structure that can be exploited for accuracy and efficiency in the ultimate application code (figure 4). This connection step must also be optimized to allow the re-generation of the mesh when changes are made to the geometry such as a deforming body or the addition of a new geometrical component. This feature is very important in order to support parameterized engineering studies where the geometry is altered in order to meet constraints or to optimize certain quantities.

As an extension of the basic Rapsodi technology, we also began development of an integrated capability to produce embedded boundary (EB) mesh geometry from general geometric descriptions. The previously available technology from NASA Ames required the user to provide a description of the geometry in terms of a water-tight surface triangulation for all embedded objects. We enhanced this capability so that EB mesh geometry can also be constructed from CAD IGES files, or by using an interactive tool for constructing geometries from simple shapes. Using Rapsodi's geometry representation also opens up the opportunity for generating higher-order EB surface information, and for dynamic generation of EB geometry during an adaptive mesh refinement-based calculation.

The technical details for the EB mesh generation capability we have developed are as follows. The CART3D package from NASA-Ames ${ }^{12}$ is used to construct embedded boundary geometry information from water-tight surface triangulations. The RAP software from the Rapsodi project [Pe02a] was modified so that surface triangulations appropriate for input into CART3D are constructed. Following the initial implementation, a new more robust topology algorithm was implemented for building water-tight surface triangulations. RAP allows the user to interactively process CAD IGES files to remove errors and de-feature the geometry in preparation for simulation and analysis (see section A). ${ }^{13}$ This now includes a capability to automatically detect and identify common geometry defects. Algorithms were developed and implemented to automatically treat singular surface patches in IGES geometry representations, further increasing the robustness of the software. RAP also provides tools for interactively constructing simple geometries, avoiding the use of complete CAD packages for cases where that level of sophistication is not needed. These new EB mesh tools are being used and further developed by members of the Rapsodi project as part of the DOE SciDAC APDEC Center. ${ }^{32}$ Figure 5 shows an example of an EB mesh constructed for a nozzle geometry, part of a study to understand the 
behavior of gas jets in an advanced high-energy accelerator concept design at Lawrence Berkeley National Laboratory.

\section{Generation of surface and volume component meshes}

Component surface and volume meshes can be generated using a number of tools. Standard algorithms involve algebraic techniques such as trans-finite interpolation, methods based on solving partial differential equations whose solution is a computational mesh, as well as geometrical construction techniques such as "marching cubes". Many of these approaches were implemented in the Overture Framework prior to the start of the Rapsodi project, and were leveraged by Rapsodi. An important prerequisite for improving on the techniques for surface generation is that the underlying CAD geometry can be evaluated easily. This issue was addressed extensively by the Rapsodi project as outlined in sections A and B above.

As part of the Rapsodi project, we developed algorithms to enhance the interactivity and automation of the embedded boundary and overset grid generation process for geometries defined by CAD descriptions. ${ }^{14,6}$ In particular, we focused on the development of tools for the interactive generation of single component meshes from CAD data. In the overset grid and multi-element grid generation process, a set of these single component meshes is combined to produce a mesh that represents the entire geometry. As discussed above, CAD descriptions in terms of IGES file formats represent a complex surface as a set of 3D trimmed NURBS patches. A typical geometry can include large regions of smooth surfaces, sharp corners, and intricate

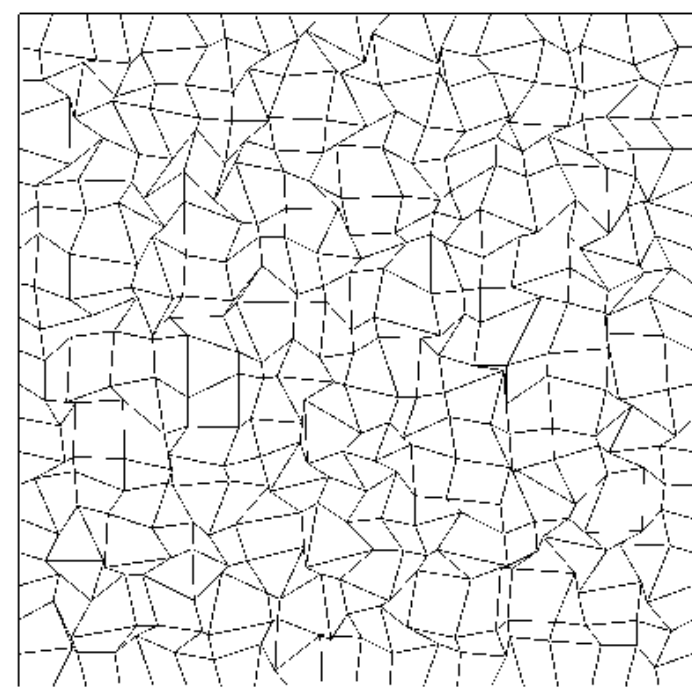

Original distorted mesh

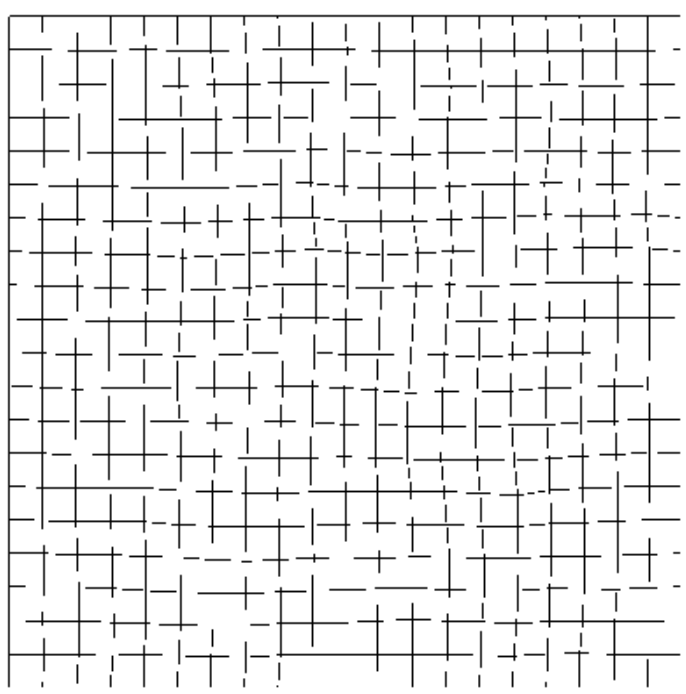

Optimized mesh

Figure 6 Example of mesh quality improvement. 
details. The representation can therefore easily have hundreds or thousands of trimmed NURBS. When generating an overset grid, one would like to construct as few surface grids as possible while faithfully representing the intricate details of the geometry. Thus, tools for automatically dealing with the trimmed NURBS representation are essential for optimal user interactivity and efficiency.

To address this need, we implemented tools for interactively inspecting the mesh topology and verifying boundary conditions on a constructed mesh. We also implemented a capability for automatically generating the background Cartesian meshes that are used to fill volumes away from boundary surfaces. Tools for interactively specifying starting curves for the hyperbolic (marching) mesh generator, including the ability to easily specify a curve that crosses multiple
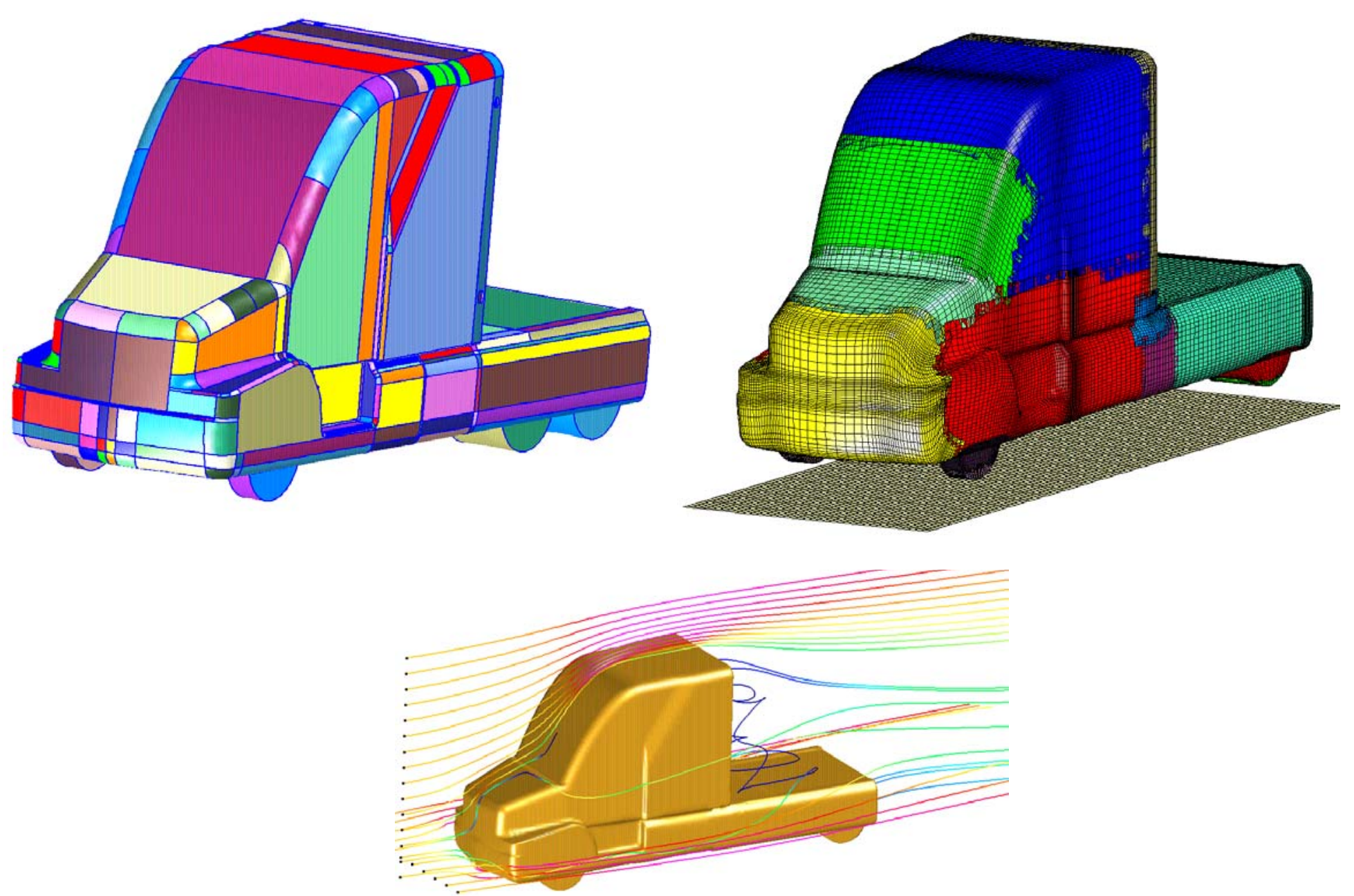

Figure 7 Rapsodi has been interacting with the Heavy Vehicle Aerodynamic Drag Project at LLNL on the analysis of truck aerodynamics. This figure shows original CAD description, volume overset mesh and flow simulation for a truck cab geometry. 
patches in a CAD description, were implemented. ${ }^{6}$ Features that help build higher-quality meshes, including ability to fit gridlines to a specified curve, and the ability to march around sharp corners in the geometry were implemented. An interactive capability for clustering grid lines for local refinement was implemented, replacing parametric (non-GUI) approach in earlier versions of the software. An enhanced ability to automatically smooth surface grids and relax grid surfaces in regions of rapid geometry variation was implemented based on the solution of elliptic equations on 3D surfaces.

\section{E. Mesh quality optimization tools}

While there is much discussion in the mesh generation community of the issue of mesh quality optimization, very little research has actually been done to understand and quantify the concepts. An important new point here is that the concept of mesh quality only makes sense in the context of a PDE that is being solved. In particular, a good mesh for one PDE might not be a good mesh for another PDE. Thus, mesh quality can be quantified in terms of the accuracy with which a PDE solution can be approximated on a given mesh. This is an extremely important mathematical issue that includes elements of a priori error estimation and sensitivity analysis.

Hyperbolic mesh generators such as the one developed in the Rapsodi project require the specification of a number of geometry-dependent parameters before the mesh can be made. To effectively use the mesh generator, the user must have a thorough understanding of how the parameters affect the resulting mesh. In fact, many random parameter combinations are invalid and prevent the mesh from being built at all. The mesh generation process can be viewed as an optimization process where the objective function is the mesh quality. We have developed a method that uses the numerical solution of a particular partial differential equation solved on the mesh as a measure of the mesh quality. Using genetic algorithms, an optimization is performed with respect to the input parameters of the mesh generator. Since the error in a numerical simulation can be made smaller simply by increasing the number of mesh points, the total number of mesh points is constrained to remain constant during the optimization process. ${ }^{15}$

Unstructured mesh optimization technology was also developed as an enhancement to the hybrid mesh generator. We have developed an optimization method based on a method of Freitag and Knupp $^{16,17}$ to improve meshes by moving vertices to "optimal" locations. Mesh quality is assessed using the properties of the Jacobian matrix of the transformation from an "ideal"' zone to an actual zone in the mesh. Analytic objective functions are built using this Jacobian and are used in a steepest descent method to move vertices to locations minimizing the objective. For example, the condition number of the Jacobian provides a measure of how much the actual zone deviates from the "shape" of the "ideal" and hence acts as an effective objective function. ${ }^{17}$ Figure 6 depicts a test case in which the optimizer corrects a highly distorted Cartesian mesh. The key feature of this method is the definition of the so-called "ideal" zone. Earlier work used equilateral triangles and tetrahedra of a specified, constant, size ${ }^{16}$. We have developed a method that allows the ideal shape to vary as needed. In the hybrid mesh generator, this information is gleaned from the stretching control procedure discussed in section C. More recently, our approach has been used to adapt a mesh to minimize the error in a numerical approximation. ${ }^{18}$ In this context, the "ideal" shape is considered to be one that resolves a feature of a PDE solution or minimizes the error in a discrete approximation. The optimization software 
has been implemented in both two dimensions and three dimensions for mixed element hybrid meshes.

\section{F. Moving or adding new geometry components}

Adding new component grids, or moving component grids during a simulation can, in principal, be very efficient with the CBM hybrid approach, an advantage for adaptive mesh or moving geometry problems. The reason for this is that the algorithms for adding a new component mesh are essentially local in nature. When a new mesh is added, its location with respect to the other meshes is determined, a new local cutout region is determined, and then a local hybrid connection mesh is constructed. Algorithms were developed in the first year of this project to make this process very fast. We continue to investigate the implications of moving geometry simulations on the mesh generation process by considering prototype simulation problems of this type.

Computational simulations involving dynamically evolving boundaries necessarily require a capability to generate and regenerate the mesh during the computation as the geometry evolves. For this reason, an efficient mesh generator is required for such simulations. As part of the Rapsodi project, we investigated the use of our mesh generation technology within the computation of viscous flow around a deforming body in two dimensions. This work, presented at the $15^{\text {th }}$ AIAA Computational Fluid Dynamics Conference in 2001, served the very valuable role of testing the robustness of the mesh generation tools developed under the Rapsodi project. ${ }^{19}$

\section{Publications and Presentations}

During the course of the LDRD project, we produced numerous new publications. In addition, all project members made presentations at conferences outside of the Laboratory. Henshaw and Fast presented their research at the Society for Industrial and Applied Mathematics Annual Meeting in Puerto Rico in July 2000. Chand presented results at the NECDEC conference in Fall of 2000 on results using A-division's HODAG code with meshes generated using Rapsodi tools. Fast and Henshaw presented results at the Fifth Symposium on Overset Composite Grid and Solution Technology at UC Davis in September, 200020. Brown presented results at the SIAM Computational Science and Engineering Conference in Washington D.C. in September, 2000 ${ }^{21}$, and also in an invited talk at the Conference on Analysis, Models and Methods at the Mathematical Sciences Research Institute in Berkeley in March 2001 ${ }^{22}$. Results were also presented by Henshaw and Petersson in a minisymposium organized by Brown on Rapid Mesh Generation at the SIAM Annual Meeting in San Diego in July 2001. ${ }^{5}$ Fast presented results at the $15^{\text {th }}$ AIAA Computational Fluid Dynamics Conference in June $2001^{19}$. Petersson, Chand and Henshaw presented papers to the 10'th International Meshing Roundtable in Newport Beach in October $2001^{4,6}$. Henshaw and Petersson presented papers to the $8^{\text {th }}$ International Conference on Numerical Grid Generation in Computational Field Simulations in June $2002^{23,13}$ and at the $6^{\text {th }}$ Overset Compsite Grid and Solution technology symposium in October $2002^{14}$. 


\section{RETURN TO THE LABORATORY}

By the end of the LDRD project, we had developed a robust code capable of reading in a CADgenerated IGES file, allowing interactive repair and cleanup of the geometry, and rapidly generating an overset, mixed-element, all-tetrahedral or embedded boundary Cartesian mesh that can be used for simulation and analysis. During the final year of the LDRD project, and continuing into FY03, we have been interacting with several projects at the Laboratory, and at other DOE facilities, that have expressed an interest in using the Rapsodi software. An interesting and unexpected result of our project was the discovery that Rapsodi's integrated code and the CAD cleanup and repair tools alone have been of interest to various projects within the Laboratory ${ }^{24}$. In particular, we have had extensive discussions with members of A-division about the possibility of incorporating Rapsodi technology into a new design for Laboratory Monte Carlo codes. Over the past year, we have been interacting with the EMSolve project, a
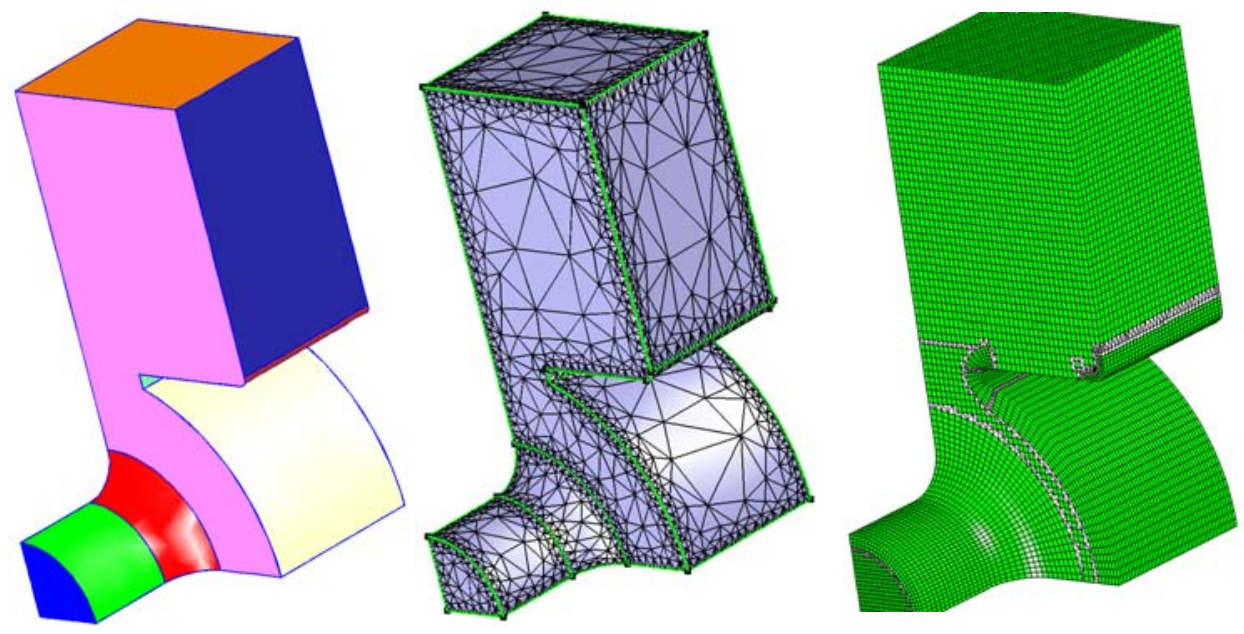

Figure 8. CAD geometry, surface triangulation and final hybrid mixed element mesh for an accelerator waveguide cavity from SLAC.

joint Engineering-Computations project to develop a finite-element based time-dependent electromagnetics solver under development as part of a LDRD Lab-Wide project. ${ }^{25}$ That project is particularly interested in our capability to generate meshes for FEM analysis that include higher order element information, required for the high-accuracy methods in their simulation code. We have generated some sample meshes for the project and provided them with a copy of one of our mesh generation codes. We have also installed codes in A-division based on Rapsodi technology. In particular, we built a custom IGES file reader that allows rapid interactive graphical inspection of complex geometries, a capability that was unavailable previously to DNT. We also installed a two-dimensional mesh generator that was requested so that some test geometries could be generated for an A-division application. 
More recently, we have been interacting with the Heavy Vehicle Aerodynamics project in Engineering and Earth and Environmental Sciences here at LLNL ${ }^{26}$ regarding issues they have for the generation of overset grids to represent the geometry of trucks (see figure 7). That project is using NASA's Overflow code for aerodynamic studies of wind resistance on large transport trucks. Rapsodi has been assisting in the mesh construction aspects of that project. We have also been in discussions with the NARAC Center at LLNL regarding future improvements to their simulation capabilities for transport and fate of aerosol releases. A future interest of that project is the ability to do high resolution fluid dynamics simulations involving unusually shaped structures, such as sports stadiums. We have been using Rapsodi technology to build meshes for such geometries. We have also been in discussion with computational scientists at the Stanford Linear Accelerator Center (SLAC) who are interested in mixed element meshes for timedependent electromagnetic field calculations (figure 8).

During FY2001, we co-authored two proposals in response to the DOE Office of Science "Scientific Discovery through Advanced Computation" (SciDAC) call for proposals. Both of these proposals were approved for funding starting in late FY2001 and are expected to bring $\$ 1099 \mathrm{~K} /$ year of new funding into the Laboratory for 5 years. The "Terascale Simulation Tools and Technology Center" 27 is a joint proposal to develop meshing and discretization technology with the CUBIT project at Sandia National Laboratory ${ }^{28}$, NWGrid at PNNL ${ }^{29}$, the FronTier project at Brookhaven National Laboratory and SUNY Stony Brook ${ }^{30}$, the MEGA ${ }^{31}$ and Trellis projects at Renssalaer Polytechnic Institute, and other collaborators with expertise in mesh generation and/or discretization technology at Argonne National Laboratory and Oak Ridge National Laboratory. A second proposal for "An Algorithmic and Software Framework for Applied Partial Differential Equations" was joint with Lawrence Berkeley National Laboratory, University of California at Davis, University of Washington, University of North Carolina, New York University and University of Wisconsin ${ }^{32}$ and includes funding for embedded boundary Cartesian mesh generation development work at LLNL as well as longer-range advanced algorithms development for embedded boundary and overset meshes. In FY2003, these two proposals are funding the use and enhancements of many of the technologies that were developed by Rapsodi with LDRD funding.

\section{THE RESEARCH TEAM}

The principal investigator, David Brown is an applied mathematician in CASC. He is an expert in numerical analysis and has extensive experience in overlapping grid generation and adaptive mesh refinement. Dr. Brown holds a Ph.D. in applied mathematics from the California Institute of Technology, a M.Sc. in geophysics and B.S. in physics from Stanford University.

William Henshaw is an applied mathematician in CASC. He has been the Overture project technical lead for the past seven years, and has seventeen years of mesh generation experience. He is also the author of the Overture mesh generator (Ogen). Dr. Henshaw holds a Ph.D. in applied mathematics from the California Institute of Technology, and a B. Math from University of Waterloo in both applied mathematics and computer science. 
N. Anders Petersson is an applied mathematician who joined CASC in October 1999. He has twelve years of experience in mesh generation research. Dr. Petersson holds a Ph. D. in numerical analysis and an M.S. in applied mathematics from the Royal Institute of Technology in Stockholm, Sweden.

Kyle Chand is computational scientist in CADSE, matrixed to CASC. He has had six years of mesh generation and applications code development experience. He holds an M.S. and B.S. in aeronautical engineering from the University of Illinois.

Petri Fast is an applied mathematician who joined CASC as a postdoctoral research fellow in August 1999. His thesis worked involved the use of overset meshes for moving boundary problems. Dr. Fast has a Ph.D. in applied mathematics from the Courant Institute of Mathematical Sciences and was a former Fulbright scholar. 


\section{REFERENCES}

${ }^{1}$ T. Michal, Computational Problem Setup, an Industrial Perspective, The Conference on High Speed Computing, April 23-26, 2001, Salishan Lodge, Gleneden Beach, Oregon.

${ }^{2}$ W. Henshaw, Ogen: An overlapping grid generator for Overture, (1999), UCRL-MA-132237.

${ }^{3}$ W. D. Henshaw, Overture: An object-oriented framework for overlapping grid applications, Paper for 2002 AIAA Conference on Applied Aerodynamics, St. Louis, MO, 2002, UCRL-JC-147889. (new publication)

${ }^{4}$ N. A. Petersson and K. Chand, Detecting errors in CAD surfaces and preparing geometries for mesh generation, presented at the $10^{\text {th }}$ International Meshing Roundtable, Newport Beach, CA, Oct. 7-10, 2001, UCRL-JC-144019-DR. (new publication)

${ }^{5}$ N. A. Petersson, Healing CAD Surfaces, abstract for minisymposium presented at SIAM annual meeting July 2001, San Diego, CA, UCRL-JC-143818-Abs. (new publication)

${ }^{6}$ W. Henshaw, An Algorithm for Projecting Points onto a Patched CAD Model, presented at the $10^{\text {th }}$ International Meshing Roundtable, Newport Beach, CA, Oct. 7-19, Engineering with Computers, 18, pp 265--273, 2002, (new publication), UCRL-JC-1440106.

7 J. Thompson, B. Soni, N. Weatherill, Handbook of Grid Generation, CRC Press LLC, 1999.

${ }^{8}$ K. Chand, Unstructured Mesh Connectivity in Unstructured Mapping, UCRL-MA-150596. (new publication)

9 K. Chand, Component-based hybrid mesh generation, Presentation to Stanford Linear Accelerator Center and LLNL A-division, UCRL-PRES-150594.

${ }^{10}$ K. Chand, Component-based hybrid mesh generation, LLNL Technical Report in preparation, 2003 (new publication)

${ }^{11} \mathrm{~K}$. Chand, Unstructured hybrid mesh support for Overture: A description of the Ugen and AdvancingFront classes and documentation for additional support classes , 2001, UCRL-MA-144020-DR (new publication).

12 M. Aftosmis, J. Melton and M. Berger, Robust and efficient Cartesian mesh generation for component-based geometry, AIAA Journal, 26 (6):952-960, 1998.

13 N. A. Petersson, A Software Demonstration of 'rap': Preparing CAD Geometries for Overlapping Grid Generation. $8^{\text {th }}$ International Conference on Numerical Grid Generation in Computational Field Simulations, June 2-6, 2002, Honolulu, HI, UCRLJC-147260. (new publication) 
${ }^{14}$ N. A. Petersson, Overlapping Grid Generation on CAD Models. $6^{\text {th }}$ Overset Compsite Grid and Solution technology symposium, October 8-10, 2002, Ft. Walton Beach, FL, UCRLPRES-149939.

${ }^{15}$ L. Carlsson and N. A. Petersson, Optimizing Chimera Grids Using Genetic Algorithms, 2001, UCRL-JC-143856 (new publication)

${ }^{16}$ L. Freitag and P.Knupp, Tetrahedral element shape optimization via the jacobian determinant and condition number, Proceedings of the $8^{\text {th }}$ International Meshing Round Table, p.247258, 1999.

${ }^{17}$ P. Knupp, Achieving finite element mesh quality via optimization of the Jacobian matrix norm and associated quantities. Part II -- A framework for volume mesh optimization and the condition number of the Jacobian matrix', International Journal for Numerical Methods in Engineering, v. 48, p. 1165-1185, 2000.

18 K. Chand, Adaptive mesh optimization using algebraic mesh quality metrics, submitted to the $4^{\text {th }}$ Symposium on Trends in Unstructured Mesh Generation, July 27-31, Albququerque, NM, UCRL-JC-151518-ABS (new publication).

${ }^{19}$ P. Fast and W. Henshaw, Time-accurate computation of viscous flow around deforming bodies using overset grids, , AIAA Paper 2001-2604, $15^{\text {th }}$ AIAA Computational Fluid Dynamics Conference, Anaheim, CA June, 2001, UCRL-JC-140714 and UCRL-VG-144407 (new publication)

20 P. Fast, Moving Overset Grid Methods for Interface Dynamics, $5^{\text {th }}$ Overset Compsite Grid and Solution technology symposium, Davis, CA, Sept 2000, UCRL-VG-140203.

21 D. L. Brown, Overture: An Object-Oriented Framework for Solving Partial Differential Equations on Overlapping Grids, UCRL-VG-138252.

${ }^{22}$ D. L. Brown, Adaptive Moving Overlapping Grids for the Solution of PDEs in Complex Geometry, UCRL-VG-143325.

23 W. D. Henshaw, Generating Composite Overlapping Grids on CAD Geometries, $8^{\text {th }}$ International Conference on Numerical Grid Generation in Computational Field Simulations, June 2-6, 2002, Honolulu, HI, UCRL-JC-147161.

$24 \mathrm{~K}$. Chand, Overture tools for geometry management and mesh generation, A-div WIP presentation, UCRL-PRES-150595.

25 D. White, Discrete time vector finite element solution of Maxwell's equations on 3D unstructured grids, UC Davis Ph.D. dissertation 1997, UCRL-LR-128238. 
26 The Heavy Vehicle Aerodynamic Drag Project, http://en-env.llnl.gov/aerodrag/ .

${ }^{27}$ D. Brown, et al, The Terascale Simulation Tools and Technologies (TSTT) Center, 2001, UCRL-PROP-142812.

${ }^{28}$ R. Leland, et. al., CUBIT Mesh Generation Environment, Technical Report SAND94-1100, Sandia National Laboratories, 1999.

${ }^{29}$ H. Trease and L. Trease, NWGrid: A Multi-Dimensional, Hybrid, Unstructured, Parallel Mesh Generation System, http://www.emsl.pnl.gov/nwgrid, 2000.

${ }^{30}$ J. Glimm, J. Grove X.-L. Li and D. C. Tan, Robust computational algorithms for dynamics interface tracking in three dimensions, SIAM J. Sci. Comp., 21, 2000.

31 M. Shephard, Meshing environment for geometry-based analysis, International Journal for Numerical Methods in Engineering, 47(1-3):169-190, 2000.

${ }^{32}$ D. Brown, et al , An Algorithmic and Software Framework for Applied Partial Differential Equations, 2001, LLNL Report UCRL-PROP-142806. 